\title{
SÍNDROME DE BURNOUT EM FISIOTERAPEUTAS INTENSIVISTAS: REVISÃO INTEGRATIVA
}

BURNOUT SYNDROME IN PHYSICAL THERAPISTS IN THE ICU: INTEGRATIVE REVIEW

SÍNDROME DE BURNOUT EN FISIOTERAPEUTAS INTENSIVISTAS: REVISIÓN INTEGRATIVA

Francisco Jairo Medeiros de Almeida ${ }^{1}$

Janaina Maria dos Santos Francisco de Paula ${ }^{2}$

Athus Bastos Brandão ${ }^{3}$

Como Citar:
Almeida FJM, De Paula JMSF, Brandão $A B$. Síndrome de burnout em fisioterapeutas intensivistas: revisão integrativa. Sanare (Sobral,

Online). 2021; 20(2):88-96.

Descritores:

Fisioterapeutas; Burnout; Esgotamento psicológico; Unidades de Cuidados Intensivos.

Descriptors:

Physical therapists; Burnout; Psychological exhaustion; Intensive Care Units.

Descriptores: Fisioterapeutas; Burnout; Agotamiento psicológico; Unidades de Cuidados Intensivos.

Submetido: $02 / 01 / 2021$

Aprovado: $17 / 11 / 2021$

Autor(a) para Correspondência: Francisco Jairo Medeiros de Almeida Escola de Saúde Pública do Ceará Rua Maria Alice Barreto Lima, 890 Coração de Jesus, Sobral-CE CEP: 62043-225 E-mail:fjmalmeida@hotmail.com

\section{RESUMO}

Este artigo tem por objetivo identificar a prevalência da síndrome de burnout em fisioterapeutas que atuam em Unidades de Terapia Intensiva no Brasil. optou-se por uma revisão integrativa da literatura, sendo realizada uma busca eletrônica entre maio e agosto de 2020, nas bases MEDLINE, LILACS, EMBASE e Index Psi - Periódicos Técnico-Científicos, com inclusão de estudos originais, publicados, disponíveis na íntegra, que foram realizados no Brasil, e excluídos aqueles que não utilizaram o Maslach Burnout Inventory como instrumento de avaliação da síndrome de burnout, validado para o português falado no Brasil, e os que não responderam aos objetivos da pesquisa. Foram incluidos quatro artigos nos quais se observou uma concordância quanto à prevalência do burnout quando utilizados os mesmos critérios de diagnóstico. $\mathrm{Na}$ presença de alteração nas três dimensões, observou-se uma variação de 3,4\% a 5,3\%, já na alteração de apenas uma dimensão, verificou-se uma variação de $31,1 \%$ a 48,2\% de presença de sindrome de burnout. Pelo critério adotado por Maslach e Jackson, o desequilíbrio nas três dimensões do burnout, a prevalência da sindrome de burnout em fisioterapeutas intensivistas brasileiros varia de $3,4 \%$ a $5,3 \%$.

\footnotetext{
1. Fisioterapeuta. Servidor Público da Prefeitura Municipal de São Gonçalo do Amarante/CE. Escola de Saúde Pública do Ceará. E-mail: fjmalmeida@hotmail.com ORCID: https://orcid.org/0000-0003-3482-8482

2. Enfermeira. Doutora em Enfermagem. Professora Adjunta do Curso de Enfermagem do Campus Amílcar Ferreira Sobral (UFPI/CAFS). Escola de Saúde Pública do Ceará. E-mail: janainasantos_upe@hotmail.com 0RCID: https:// orcid.org/0000-0002-5898-3201

3. Fisioterapeuta. Especialista em Osteopatia Clínica. Escola de Saúde Pública do Ceará. E-mail: athusbrandao@ gmail.com ORCID: https://orcid.org/0000-0002-4767-5983
}

Cert. de Redação Científica: Central das Revisões. Edição de texto: Karina Maria M. Machado. Revisão de provas: Texto definitivo validado pelos(as) autores(as). 


\section{ABSTRACT}

This article aims to identify the prevalence of Burnout Syndrome in Physical Therapists working in Intensive Care Units in Brazil. We chose to perform an integrative literature review with electronic search between May and August 2020, in databases MEDLINE, LILACS, EMBASE e Index Psi-Periódicos Técnico-Cientificos, with the inclusion of original studies, published and available in full, carried out in Brazil, and with the exclusion of those that did not use the Maslach Burnout Inventory, validated for Brazilian Portuguese, as an assessment tool for Burnout Syndrome and, also, of those that were not pertinent to the research objectives. Four articles were included in which an agreement was observed regarding the prevalence of burnout when using the same diagnostic criteria. In the presence of changes in the three dimensions, we observed that the Burnout Syndrome varied from $3.4 \%$ to $5.3 \%$, while in the presence of changes in only one dimension, the variation went from $31.1 \%$ to $48.2 \%$. According to the criterion adopted by Maslach and Jackson, the imbalance in all three burnout dimensions, the prevalence of Burnout Syndrome in Brazilian Physical therapists working in ICUs varies from $3.4 \%$ to $5.3 \%$.

\section{RESUMEN}

Este artículo tiene como objetivo identificar la prevalencia de sindrome de burnout en Fisioterapeutas que actúan en Unidades de Terapia Intensivista en Brasil. Se eligió por revisión integrativa de la literatura, siendo realizada una búsqueda electrónica entre mayo y agosto de 2020, en las bases MEDLINE, LILACS, EMBASE e Index Psi-Periódicos Técnico-Científicos, con inclusión de estudios originales, publicados, disponibles integralmente, realizados en Brasil, y rechazados aquellos que no utilizaron el Maslach Burnout Inventory como herramienta de evaluación de la sindrome de burnout, validado para el portugués de Brasil, y los que no respondieron a los objetivos de la investigación. Fueron añadidos cuatro artículos los cuales se observó una concordancia cuanto a la prevalencia del burnout cuando utilizados los mismos criterios de diagnóstico. En la presencia de la alteración en las tres dimensiones, observamos una variación de 3,4\% a 5,3\%, ya en la alteración de apenas una dimensión, verificamos una variación de $31,1 \%$ a $48,2 \%$ de presencia de sindrome de burnout. Por el criterio adoptado por Maslach y Jackson, el desequilibrio en las tres dimensiones de burnout, la prevalencia de la Sindrome en fisioterapeutas intensivistas brasileños está entre $3.4 \%$ a 5,3\%.

\section{INTRODUÇÃO}

0 conceito de sindrome de burnout (SB) foi criado nos anos 1970, para nomear uma reação de esgotamento físico e mental vivenciada por profissionais de saúde envolvidos na assistência a usuários de drogas ${ }^{1}$.

Ela é descrita por meio de três dimensões: Exaustão Emocional (EE), Despersonalização (DP) e Realização Profissional (RP) ${ }^{2}$. A EE é caracterizada pela ausência ou déficit de energia associada à sensação de que todos os recursos emocionais e físicos se esgotaram; a DP apresenta-se como a perda da sensibilidade emocional associada a reações negativas e impiedosas direcionadas àqueles para os quais os serviços são prestados; e a RP caracteriza-se por autoavaliação negativa por parte do profissional sobre o seu desempenho pessoal e no ambiente de trabalho ${ }^{3}$.

0 Maslach Burnout Inventory (MBI), instrumento de investigação mais utilizado na literatura para a detecção da SB, foi elaborado por Christina Maslach e Susan Jackson, traduzido e validado para o português falado no Brasil por Lautert em $1995^{4}$.

0 surgimento do MBI e a tradução deste para vários idiomas, além de permitir o desenvolvimento da pesquisa epidemiológica, estendeu esse conceito a diversos países e amostras populacionais, ampliando a detecção do agravo em outras ocupações fora das tradicionais áreas de saúde. Dessa forma, a percepção de que a organização do trabalho e a relação com o trabalho em si, não necessariamente as atividades específicas, são consideradas seus principais determinantes, implica aos gestores a adoção de medidas que promovam a saúde mental no ambiente laboral como forma de mitigação deste agravo ${ }^{1}$.

Criada a partir da necessidade de aperfeiçoamento para o atendimento a pacientes em estado crítico, as Unidades de Terapia Intensiva (UTI) configuramse como um dos ambientes mais agressivos, sobrecarregados e exaustivos do hospital, é um local onde a tensão e a ocorrência de óbitos são constantes, onde os profissionais necessitam estar em alerta permanente para qualquer situação de emergência e estão expostos a ruídos excessivos, 
rotinas rígidas e inflexiveis. Profissionais que trabalham nesses ambientes, dentre eles, o fisioterapeuta, estão propensos ao desenvolvimento de sofrimento psíquico, dada a complexidade das ações ali realizadas, bem como o estresse gerado durante sua realização ${ }^{5}$.

A sindrome de burnout trata-se de uma condição de saúde que afeta de maneira significativa a qualidade do atendimento, que pode estar associada a elevados índices de absenteísmo e ao aumento de erros nas condutas profissionais. 0 entendimento sobre sua prevalência poderá fornecer indícios para que os gestores implantem medidas de adoção de um ambiente laboral mais saudável. Assim, justifica-se a realização desta pesquisa.

0 objetivo deste estudo é identificar a prevalência da sindrome de burnout em fisioterapeutas que atuam em Unidades de Terapia Intensiva no Brasil.

\section{METODOLOGIA}

0 presente estudo se trata de uma revisão integrativa da literatura em que a busca eletrônica, elaborada com base nos critérios estabelecidos pelo Cochrane Handbook, incluiu estudos originais, publicados, disponíveis na integra e que foram realizados no Brasil, e excluídos aqueles que não utilizaram o Maslach Burnout Inventory (MBI) como instrumento de avaliação para a síndrome de burnout validado para o português falado no Brasil e os que não responderam aos objetivos da pesquisa; não houve delimitação de ano ou idioma na seleção dos estudos ${ }^{6}$.
Por tratar-se de uma condição de saúde que sofre influência de fatores individuais, sociais e laborais, optou-se por restringir a estudos realizados apenas no Brasil, haja vista as diferenças no contexto de trabalho e/ou sociais, pelas quais os profissionais estudados são submetidos em outras realidades.

A pergunta norteadora foi desenvolvida por meio da estratégia PICo, acrônimo para as palavras "População, Interesse e Contexto", e permitiu que a equipe de pesquisadores, na fase de construção, combinasse os descritores controlados e não controlados, possibilitando as respostas apropriadas aos questionamentos e identificando a melhor evidência científica sobre o tema ${ }^{7}$.

Portanto, adotou-se a seguinte pergunta norteadora: Qual a prevalência da síndrome de burnout em fisioterapeutas que atuam em Unidades de Terapia Intensiva no Brasil?

Os dados foram coletados nos meses de maio a agosto de 2020, por intermédio do Portal de Periódicos Capes, com acesso pelo sistema da Comunidade Acadêmica Federada - Universidade Federal do Piauí (CAFe/UFPI), nas bases da Medical Literature Analysis and Retrieval System Online (MEDLINE), Literatura Latino-americana e do Caribe em Ciências da Saúde (LILACS), Excerpta Medica dataBASE (EMBASE) e Index Psicología - Periódicos técnico-científicos.

A Figura 1 ilustra os descritores controlados de dados obtidos por meio de consulta aos Descritores em Ciências da Saúde (DeCS), Medical Subject Headings (MeSH), Terminologia Psi e Emtree thesaurus, a partir do acrônimo PICo.

Figura 1. Levantamento dos termos a partir dos descritores DeCS/MeSH/Terminologia Psi e Emtree thesaurus.

\begin{tabular}{|c|c|l|}
\hline \multicolumn{2}{|c|}{ Descritores em Ciências da Saúde - DeCS } \\
\hline P & $D C$ & $\begin{array}{l}\text { Physical Therapists/Fisioterapeutas/Fisioterapeutas/Physical Therapist Assistants/ } \\
\text { Asistentes de Fisioterapeutas/Assistentes de Fisioterapeutas }\end{array}$ \\
\hline I & $D C$ & $\begin{array}{l}\text { Burnout, Psychological/AgotamientoPsicológico/Esgotamento Psicológico/Depersonalization } \\
\text { Despersonalización/Despersonalização/Work Engagement/Compromiso Laboral/Engajamento } \\
\text { no Trabalho/Burnout, Professional/Agotamiento Profesional/Esgotamento Profissional/ } \\
\text { Compassion Fatigue/Desgaste por Empatía/Fadiga por Compaixão/Occupational Stress/Estrés } \\
\text { Laboral/Estresse Ocupacional }\end{array}$ \\
\hline Co & $D C$ & $\begin{array}{l}\text { Intensive Care Units/Unidades de Cuidados Intensivos/Unidades de Terapia Intensiva/ } \\
\text { Intensive Care Units, Pediatric/Unidades de Cuidado Intensivo Pediátrico/Unidades de } \\
\text { Terapia Intensiva Pediátrica/Intensive Care Units, Neonatal/Unidades de Cuidado Intensivo } \\
\text { Neonatal/Unidades de Terapia Intensiva Neonatal/Critical Care/Cuidados Críticos/Cuidados } \\
\text { Críticos }\end{array}$ \\
\hline
\end{tabular}




\begin{tabular}{|c|c|c|}
\hline \multicolumn{3}{|c|}{ Medical Subject Headings - MeSH } \\
\hline $\mathrm{P}$ & DC & Physical Therapists \\
\hline I & DC & Burnout, Psychological/Burnout, Professional/Stress, Psychological \\
\hline Co & DC & $\begin{array}{l}\text { Intensive Care Units/Intensive Care Units, Pediatric/Intensive Care Units, Neonatal/Critical } \\
\text { Care }\end{array}$ \\
\hline \multicolumn{3}{|c|}{ Terminologia Psi } \\
\hline $\mathrm{P}$ & $D C$ & Terapeutas/Therapists/Terapéutas/Fisioterapeutas/Phisycal Therapists/Fisioterapeutas \\
\hline I & $D C$ & $\begin{array}{l}\text { Stress Ocupacional/Occupational Stress/Estres Ocupacional/Burnout/Despersonalização/ } \\
\text { Depersonalization/Despersonalización }\end{array}$ \\
\hline Co & DC & UTI/Unidades de Terapia Intensiva/Intensive Care Units/Unidades de Terapia Intensiva/CTI \\
\hline \multicolumn{3}{|c|}{ Emtree thesaurus } \\
\hline$P$ & $D C$ & Physiotherapist/respiratory Therapist \\
\hline I & $D C$ & Burnout/professional Burnout/compassion fatigue/job stress/emotional stress \\
\hline Co & $D C$ & Intensive care unit/neonatal intensive care unit/pediatric intensive care unit/intensive care \\
\hline
\end{tabular}

* DC: Descritores controlados.

Fonte: Dados da pesquisa.

Para guiar a redação do protocolo e do resultado final da revisão, utilizou-se o Checklist Preferred Items for Systematic Reviews and Meta-Analyses (PRISMA), que é composto por 27 tópicos para assegurar o desenvolvimento adequado da pesquisa, utilizando-se o fluxograma para a discriminação das etapas de seleção dos artigos incluídos ${ }^{8}$.

A seleção dos estudos foi realizada de forma independente por dois pesquisadores a partir da leitura dos títulos e resumos de cada artigo. Foram eliminados numa primeira etapa aqueles que claramente não se enquadraram nos critérios de elegibilidade, os aprovados foram lidos na íntegra para ratificar sua inclusão ou não na pesquisa. Na ocorrência de discordâncias, estas foram resolvidas por consenso. A qualidade dos estudos foi avaliada pela Critical Appraisal Checklist for Prevalence Studies ${ }^{9}$.

Os dados foram extraídos a partir de um formulário elaborado pelos autores e organizados em tabelas, contemplando as características dos estudos incluídos (autoria, ano de publicação, desenho do estudo, número de amostra, local da pesquisa), critérios de diagnóstico, prevalência e percentual dos domínios da SB. As discordâncias foram resolvidas por consenso ou por um terceiro revisor independente.

A análise de dados foi realizada por meio de uma síntese descritiva com análise qualitativa.

\section{RESULTADOS}

A busca identificou 126 estudos nas bases de dados MEDLINE, LILACS, EMBASE e Index Psicología - Periódicos técnico-científicos. Após a aplicação dos critérios de elegibilidade, foram incluídos quatro artigos, conforme detalhado no fluxograma de discriminação das etapas de seleção dos artigos ilustrado na Figura 2. 
Figura 2. Fluxograma de discriminação das etapas de seleção dos artigos.

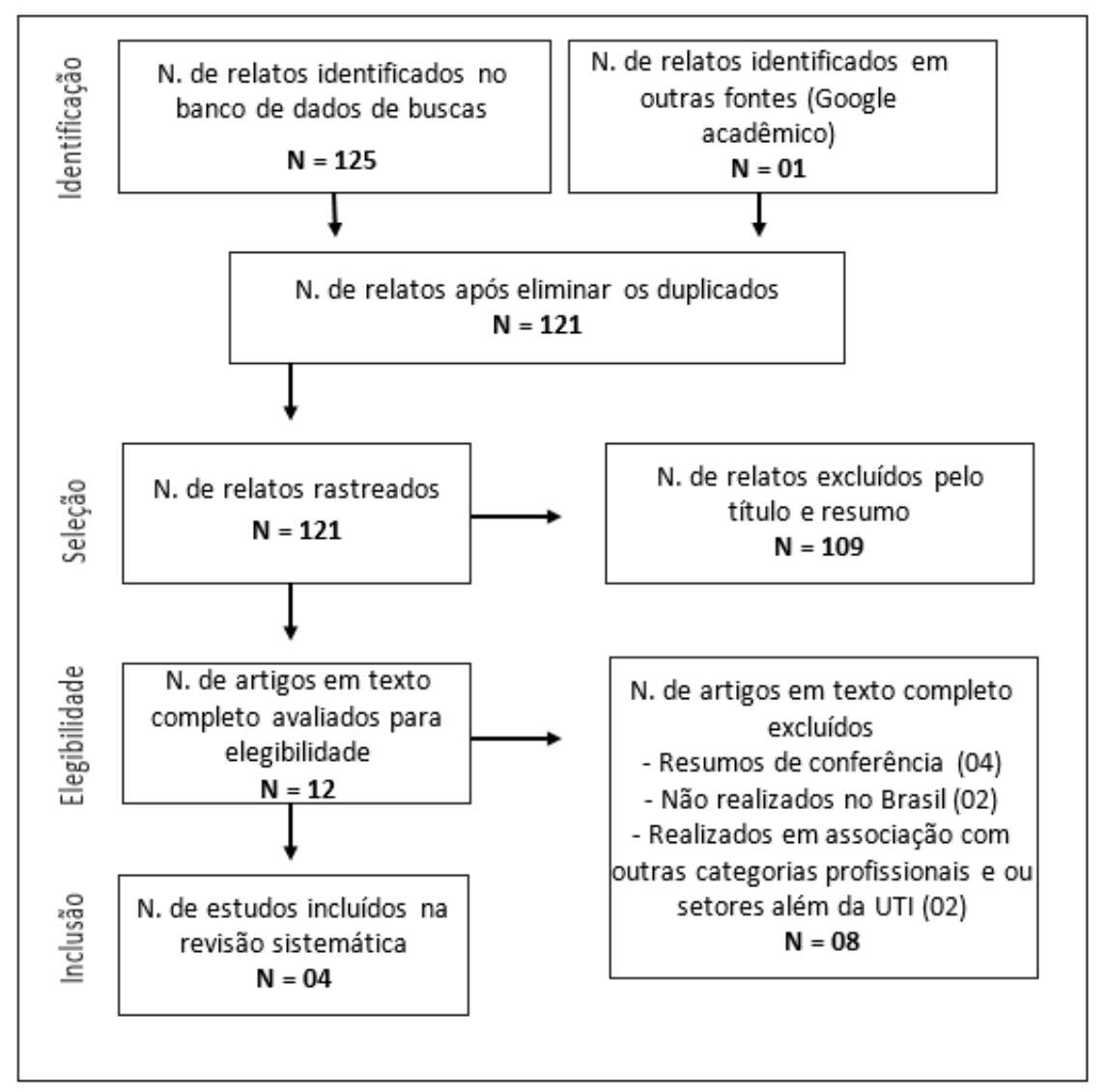

Fonte: Dados da pesquisa.

0 Quadro 1 traz as características dos estudos incluídos e os achados referentes às dimensões da síndrome de burnout.

Quadro 1. Características dos estudos incluídos na revisão.

\begin{tabular}{|c|c|c|c|c|}
\hline \multicolumn{5}{|c|}{ Caracterização geral dos estudos } \\
\hline Autores & Nascimento et al. & Rosa et al. & Santos et al. & Silva et al. \\
\hline Ano & 2017 & 2018 & 2018 & 2018 \\
\hline Local & $\begin{array}{l}\text { UTI em Vitoria da } \\
\text { Conquista-BA }\end{array}$ & $\begin{array}{l}5 \text { hospitais } \\
\text { Salvador-BA }\end{array}$ & $\begin{array}{c}7 \text { hospitais Feira } \\
\text { de Santana-BA }\end{array}$ & $\begin{array}{l}5 \text { hospitais } \\
\text { Recife-PE }\end{array}$ \\
\hline Tipo de estudo & Transversal & Transversal & Transversal & Transversal \\
\hline Número da amostra & 25 & 45 & 60 & 56 \\
\hline \multicolumn{5}{|c|}{ Percentual das dimensões da sindrome de burnout } \\
\hline Exaustão Emocional (EE) & $\begin{array}{l}\text { Alta } 12,0 \% \\
\text { Média } 36,0 \% \\
\text { Baixa } 52,0 \%\end{array}$ & $\begin{array}{l}\text { Alta } 20,0 \% \\
\text { Média } 35,6 \% \\
\text { Baixa } 44,4 \%\end{array}$ & $\begin{array}{l}\text { Alta } 38,3 \% \\
\text { Média } 31,7 \% \\
\text { Baixa } 30,0 \%\end{array}$ & $\begin{array}{l}\text { Alta } 58,9 \% \\
\text { Média } 32,2 \% \\
\text { Baixa } 08,9 \%\end{array}$ \\
\hline Despersonalização (DP) & $\begin{array}{l}\text { Alta } 08,0 \% \\
\text { Média } 28,0 \% \\
\text { Baixa } 64,0 \%\end{array}$ & $\begin{array}{l}\text { Alta } 02,2 \% \\
\text { Média } 20,0 \% \\
\text { Baixa } 77,8 \%\end{array}$ & $\begin{array}{l}\text { Alta } 16,7 \% \\
\text { Média } 11,7 \% \\
\text { Baixa } 71,7 \%\end{array}$ & $\begin{array}{c}\text { Alta } 17,9 \% \\
\text { Média } 26,8 \% \\
\text { Baixa } 55,3 \%\end{array}$ \\
\hline Realização Profissional (RP) & $\begin{array}{l}\text { Alta } 76,0 \% \\
\text { Média } 04,0 \% \\
\text { Baixa } 20,0 \%\end{array}$ & $\begin{array}{l}\text { Alta } 13,3 \% \\
\text { Média } 22,2 \% \\
\text { Baixa } 64,4 \%\end{array}$ & $\begin{array}{l}\text { Alta } 15,0 \% \\
\text { Média } 40,0 \% \\
\text { Baixa } 45,0 \%\end{array}$ & $\begin{array}{c}\text { Alta } 37,5 \% \\
\text { Média } 34,0 \% \\
\text { Baixa } 28,5 \%\end{array}$ \\
\hline
\end{tabular}

Fonte: Dados da pesquisa. 
Os estudos foram realizados no Nordeste brasileiro, sendo três deles conduzidos em hospitais do estado da Bahia, sua totalidade foi composta por estudos de corte transversal. A amostra dos estudos foi de 186 indivíduos, sendo o sexo feminino o que mais se destacou dentro da amostra, 107 (57,5\%).

Em se tratando das dimensões do burnout, que são classificadas em alta, média e baixa, a partir de pontos de corte predefinidos, observou-se uma variação para o nível alto de EE de $12,0 \%$ a 58,8\%; nível alto de DP de $02,2 \%$ a $17,9 \%$; e nível baixo de RP de $20,0 \%$ a $64,4 \%$ entre os estudos.

0 Quadro 2 apresenta a prevalência e critérios de diagnóstico da SB.

Quadro 2. Prevalência e critérios de diagnóstico da síndrome de burnout.

\begin{tabular}{|l|c|c|}
\hline Estudo & Critérios de diagnóstico da síndrome de burnout & Síndrome de burnout \\
\hline $\begin{array}{l}\text { Rosa } \\
\text { et al. }{ }^{10}\end{array}$ & $\begin{array}{c}\text { Desequilíbrio em pelo menos uma dimensão. Nível alto de EE } \\
(\geq 27 \text { pontos) ou DP }(\geq 13 \text { pontos) ou baixo na RP ( } \leq 31 \text { pontos) }\end{array}$ & 01 dimensão: 14 (31,1\%) \\
\hline $\begin{array}{l}\text { Nascimento } \\
\text { et al. }{ }^{11}\end{array}$ & $\begin{array}{c}\text { Nível alto nas dimensões EE ( } \geq 26 \text { pontos) e DP ( } \geq 13 \text { pontos) e } \\
\text { baixo na RP ( } \leq 31 \text { pontos) }\end{array}$ & 03 dimensões: 01 (04\%) \\
\hline $\begin{array}{l}\text { Silva } \\
\text { et al. }{ }^{12}\end{array}$ & $\begin{array}{c}\text { Desequilíbrio em três ou pelo menos uma dimensão. Nível alto } \\
\text { de EE ( } \geq 27 \text { pontos) e/ou DP ( } \geq 13 \text { pontos) e/ou baixo na RP } \\
(\leq 31 \text { pontos) }\end{array}$ & $\begin{array}{c}01 \text { dimensão: } 27(48,2 \%) \\
03 \text { dimensões: } 03(5,3 \%)\end{array}$ \\
$\begin{array}{l}\text { Santos } \\
\text { et al. }{ }^{13}\end{array}$ & $\begin{array}{c}\text { Desequilíbrio em três ou pelo menos uma dimensão. Nível alto } \\
\text { de EE ( } \geq 27 \text { pontos) e/ou DP ( } \geq 13 \text { pontos) e/ou baixo na RP } \\
(\leq 31 \text { pontos) }\end{array}$ & $\begin{array}{c}01 \text { dimensão: } 20(33,3 \%) \\
03 \text { dimensões: } 02(3,4 \%)\end{array}$ \\
\hline
\end{tabular}

EE: Exaustão Emocional; DP: Despersonalização; RP: Realização Profissional.

Fonte: Dados da pesquisa.

Quanto aos aspectos relacionados à SB, foi possivel observar que, embora as idealizadoras do instrumento MBI configurem o diagnóstico de burnout apenas na presença de desequilíbrio nas suas três dimensões, não houve um consenso quanto aos critérios utilizados para a constatação da presença desta; dessa forma, o estudo de Rosa et al. ${ }^{10}$ tomou como referência o desequilíbrio em apenas uma dimensão, Nascimento et al. ${ }^{11}$ considerou o desequilíbrio nas três dimensões, já Silva et al. ${ }^{12}$ e Santos et al. ${ }^{13}$ tomaram como base os dois métodos citados anteriormente. Observa-se que nos estudos selecionados há uma concordância quanto à prevalência do burnout quando utilizados os mesmos critérios de diagnóstico. Na presença de alteração nas três dimensões, existe uma variação de 3,4\% a 5,3\%, já na alteração de apenas uma dimensão, verifica-se uma variação de $31,1 \%$ a $48,2 \%$ de presença de SB.

A qualidade dos estudos selecionados, realizada através da Critical Appraisal Checklist for Prevalence Studies, está descrita no Quadro 3.

Quadro 3. Avaliação da qualidade dos estudos.

\begin{tabular}{|l|c|c|c|c|c|c|c|c|c|c|}
\hline Estudos & $\mathbf{1}$ & $\mathbf{2}$ & $\mathbf{3}$ & $\mathbf{4}$ & $\mathbf{5}$ & $\mathbf{6}$ & $\mathbf{7}$ & $\mathbf{8}$ & $\mathbf{9}$ & \multicolumn{2}{c|}{ Score } \\
\hline Nascimento et al. ${ }^{11}$ & $\mathrm{Y}$ & $\mathrm{Y}$ & $\mathrm{N}$ & $\mathrm{Y}$ & $\mathrm{Y}$ & $\mathrm{Y}$ & $\mathrm{Y}$ & $\mathrm{Y}$ & $\mathrm{Y}$ & 08 \\
\hline Rosa et al. $^{10}$ & $\mathrm{Y}$ & $\mathrm{Y}$ & $\mathrm{U}$ & $\mathrm{Y}$ & $\mathrm{Y}$ & $\mathrm{N}$ & $\mathrm{Y}$ & $\mathrm{Y}$ & $\mathrm{Y}$ & 07 \\
\hline Silva et al.12 & $\mathrm{Y}$ & $\mathrm{Y}$ & $\mathrm{N}$ & $\mathrm{Y}$ & $\mathrm{Y}$ & $\mathrm{Y}$ & $\mathrm{Y}$ & $\mathrm{Y}$ & $\mathrm{Y}$ & 08 \\
\hline Santos et al.13 & $\mathrm{Y}$ & $\mathrm{Y}$ & $\mathrm{Y}$ & $\mathrm{Y}$ & $\mathrm{Y}$ & $\mathrm{Y}$ & $\mathrm{Y}$ & $\mathrm{Y}$ & $\mathrm{Y}$ & 09 \\
\hline
\end{tabular}

1. Was the sample frame appropriate to address the target population? 2. Were study participants sampled in an appropriate way? 3. Was the sample size adequate? 4. Were the study subjects and the setting described in detail? 5. Was the data analysis conducted with sufficient coverage of the identified sample? 6 . Were valid methods used for the identification of the condition? 7. Was the condition measured in a standard, reliable way for all participants? 8. Was there appropriate statistical analysis? 9. Was the response rate adequate, and if not, was the low response rate managed appropriately?

$\mathrm{Y}=$ yes; $\mathrm{N}=$ no; $\mathrm{U}=$ unclear

Fonte: Dados da pesquisa. 
A escala Critical Appraisal Checklist for Prevalence Studies pontua a qualidade dos estudos de prevalência em uma escala de 0 a 9 pontos; os estudos selecionados apresentaram pontuação de 7 a 9. 0s itens não pontuados foram: o 3, que questiona sobre o tamanho da amostra - nesse quesito, apenas o estudo de Santos et al. ${ }^{13}$ trouxe uma amostra adequada para um nível de confiança de $95 \%$ e uma margem de erro de 0,05 ; e o item 6, que questiona sobre a utilização de métodos válidos para a identificação da doença - neste, apenas o estudo de Rosa et al. ${ }^{10}$ não cumpriu o requisito, pois, embora tenha utilizado o MBI como instrumento, os autores não realizaram a interpretação do mesmo seguindo os critérios propostos pelo estudo que validou o MBI para a população brasileira.

\section{DISCUSSÃO}

Pela falta de consenso na avaliação e diagnóstico da SB por meio do MBI entre os estudos selecionados, a discussão será realizada considerando os autores que utilizaram os mesmos critérios entre si. No entanto, vale salientar que Maslach e Jackson ${ }^{14}$, as idealizadoras do MBI, consideram o diagnóstico da SB na presença de disfunção nas três dimensões, ou seja, grau alto nas dimensões de EE e DP e baixo na RP para a determinação do burnout.

Existem relatos na literatura quanto à falta de um consenso literário a respeito dos critérios diagnósticos para SB; Barros et al. ${ }^{15}$ observaram que tanto os valores conceituais utilizados como referência quanto as características populacionais podem justificar a variação da prevalência de SB tão presentes nos estudos ${ }^{15}$.

Os autores Rosa et al. ${ }^{10}$, Silva et al. ${ }^{12}$ e Santos et al. ${ }^{13}$ consideraram para o diagnóstico da SB 0 desequilíbrio em pelo menos uma dimensão, eles obtiveram como prevalência 14/31,1\%, 20/33,3\% e $27 / 48,2 \%$, respectivamente, valores esses muito abaixo dos encontrados em outro estudo ${ }^{3}$ que obteve $62,9 \%$ de prevalência de SB em uma amostra de 27 fisioterapeutas intensivistas de um hospital de alta complexidade da cidade de Recife. Essa divergência pode ser justificada pela diferença nos pontos de corte das dimensões de DP e RP que foram diferentes em relação aos estudos selecionados, estes consideraram pontuações $\geq 10$ para DP como nível alto e $\leq 33$ para RP como nível baixo; já aqueles $\geq 13$ para DP como nível alto e $\leq 31$ para RP como nível baixo. 0s autores Rosa et al. ${ }^{11}$, Silva et al. ${ }^{12}$ e Santos et al. ${ }^{13}$ obtiveram nos seus estudos uma prevalência de SB de $01 / 4,0 \%, 03 / 3,4 \%$ e $03 / 5,3 \%$, respectivamente; eles consideraram 0 desequilíbrio nas três dimensões. Resultados similares a esses puderam ser observados no estudo de Ferreira et al. ${ }^{16}$, que observaram que 3 fisioterapeutas estiveram dentro dos critérios de diagnóstico de SB em uma amostra de 65 profissionais intensivistas que atuavam em um hospital de referência da cidade de Fortaleza. Salienta-se que, em sua pesquisa, estavam inclusas outras categorias profissionais.

É importante destacar que variáveis sociodemográficas não são preditivas da SB, elas funcionam apenas como facilitadoras ou inibidoras, já que o burnout não é caracterizado por um problema pessoal e exclusivo do trabalhador, e sim do ambiente social onde este se encontra inserido ${ }^{17}$.

A respeito dos percentuais obtidos em cada dimensão de forma isolada, a discussão pontuará as alterações que levam à ocorrência da SB, ou seja, escore alto em EE e DP e baixo na RP. Foi observada uma variação considerável entre os estudos e, embora os pontos de corte entre os autores tenham sido os mesmos, a EE apresentou valores de $12,0 \%$ a $58,9 \%$, a DP de $2,2 \%$ a $17,9 \%$ e RP de $20,0 \%$ a $64,4 \%$. Em um estudo realizado por Fumis et al. ${ }^{18}$, observouse uma prevalência de $50,0 \%, 41,0 \%$ e $59,0 \%$ de $E E$, DP e RP, respectivamente, em uma amostra de 57 fisioterapeutas - valores esses dentro da variação encontrada nos estudos selecionados, exceto para a $D P$, que alcançou valores maiores.

Em outro estudo ${ }^{3}$, que teve como objetivo caracterizar a presença da SB nos fisioterapeutas de um hospital público de alta complexidade e verificar uma possivel relação da síndrome com variáveis demográficas e laborais, obteve-se uma prevalência de alta EE e DP com valores de $35,4 \%$ e $29,2 \%$, respectivamente, e baixa RP, com $6,3 \%$.

Estudos nos mostram que o número excessivo de pacientes está relacionado a sintomas depressivos em profissionais de saúde ${ }^{2}$, além de elevados níveis de EE em unidades com maiores taxas de fatalidade, número elevado de óbitos e elevada proporção de pacientes idosos. Os aspectos organizacionais como o número insuficiente de pessoal, longas jornadas de trabalho, a falta de reconhecimento profissional, o funcionamento da equipe e o estilo de liderança das chefias são fatores importantes na ocorrência de $E^{19}$.

Nos fisioterapeutas, a resposta crônica à tensão 
emotiva do exercício profissional e aos cuidados envolvidos com seres humanos é considerada um tipo de tensão do trabalho, que é criada entre o terapeuta e o paciente. Ao diminuir a emoção sentida durante o trabalho, é desencadeado um processo de despersonalização do profissional, definido como um estado no qual não há mais compaixão, respeito ou pensamentos positivos do profissional para com o paciente; dessa forma, desenvolve-se uma avaliação negativa referente ao seu local de trabalho ${ }^{16}$.

\section{CONCLUSÃO}

Pelo critério adotado por Maslach e Jackson, ou seja, o desequilíbrio nas três dimensões do burnout, a prevalência da síndrome de burnout em fisioterapeutas intensivistas brasileiros varia de $3,4 \%$ a $5,3 \%$.

Os estudos que avaliam a presença da SB em fisioterapeutas intensivistas no Brasil ainda são muito escassos, pontuais e com amostras muito pequenas, o que dificulta a comparação entre outros estudos nacionais.

Outro fator fundamental é a falta de consenso e critérios no método de avaliação da SB, bem como dos pontos de corte nas suas dimensões, isso acaba trazendo resultados muito heterogêneos, sendo assim, abre-se uma oportunidade para alavancar mais pesquisas a serem realizadas na área em questão, adequando e tornando o desenho metodológico de modo mais específico, a fim de homogeneizar os achados entre os pesquisadores.

\section{CONTRIBUIÇÃO DOS AUTORES}

Francisco Jairo Medeiros de Almeida contribuiu com o delineamento, a realização da pesquisa e a redação do manuscrito. Janaina Maria dos Santos Francisco de Paula contribuiu com a orientação, o delineamento da pesquisa e a revisão crítica do manuscrito. Athus Bastos Brandão contribuiu com o delineamento da pesquisa, a realização da pesquisa e a redação do manuscrito.

\section{REFERÊNCIAS}

1. Vieira I, Russo JA. Burnout e estresse: entre medicalização e psicologização. Physis [serial on the internet]. 2019 [cited 2020 Apr 11]; 29(2):1-22. Avaliable from http://www. scielo.br/scielo.php?script=sci arttext\&pid $=$ S0103-73312019000200604
2. Ferreira GB, Aragão AEA, Oliveira PS. Síndrome de burnout na enfermagem hospitalar/intensivista: o que dizem os estudos? Sanare (Sobral, Online) [serial on the internet]. 2017 [cited 2020 Apr 11]; $16(1): 100$ 8. Avaliable from: https://sanare.emnuvens.com.br/ sanare/article/view/1100

3. Santos ER, Neri, LV, Wanderley ELS. Síndrome de Burnout em fisioterapeutas de um hospital público de alta complexidade da cidade do Recife, Pernambuco. Acta Fisiatr [serial on the internet]. 2018 [cited 2020 Apr 11]; 250(1):315. Avaliable form: http://www.revistas.usp.br/ actafisiatrica/article/view/158832

4. Zanatta AB, Lucca SR. Prevalência da síndrome de Burnout em profissionais da saúde de um hospital oncohematológico infantil. Rev Esc Enferm USP [serial on the internet]. 2015 [cited 2020 May 20]; 49(2):253-60. Avaliable from: https:// www.scielo.br/pdf/reeusp/v49n2/pt 0080-6234reeusp-49-02-0253.pdf

5. Cruz SP, Abellan MV. Desgaste profissional, stress e satisfação no trabalho do pessoal de enfermagem em um hospital universitário. Rev Lat-Am Enferm [serial on internet]. 2015 [cited 2020 May 20]; 23(3):543-52. Avaliable from: https://www.scielo.br/j/rlae/a/Xn3jBc6T sNwMf3fTXb9JJBd/? lang =pt

6. Donato H, Donato M. Etapas na condução de uma revisão sistemática. Acta Med Port [serial on the internet]. 2019 [cited 2020 Nov 20]; 32(3):22735. Avaliable from: https://www.researchgate .net/publication/332084935 Etapas na Conducao de uma Revisao Sistematica

7. Araújo WCO. Recuperação da informação em saúde: construção, modelos e estratégias. ConCi: Com Ciênc Inform [serial on the internet]. 2020 [cited 2020 May 20];3(2):100-34. Avaliable from: https://seer. ufs.br/index.php/conci/article/view/13447

8. Galvão TF, Pansani ASA, Harrad D. Principais itens para relatar Revisões sistemáticas e Meta-análises: A recomendação PRISMA. Epidemiol Serv Saúde [serial on the internet]. 2015 [cited 2020 Apr 12]; 24(2):33542. Avaliable from: http://www.scielo.br/scielo.php? script $=$ sci arttext \&pid=S2237-96222015000200335

9. Munn Z, Moola S, Lisy K, Riitano D, Tufanaru C. Methodological guidance for systematic reviews of observational epidemiological studies reporting prevalence and cumulative incidence data. Int J Evid Based Healthc [serial on the internet]. 2015 [cited 2020 Nov 20]; 13(3):147-53. Avaliable from: https:// pubmed.ncbi.nlm.nih.gov/26317388/

10. Rosa FWC, Silva TB, Maciel RRB, Portella DDA, Duarte ACM, Mercês MC. Frequência da Síndrome de Burnout em uma amostra de fisioterapeutas intensivistas. Rev Pesq e Fisio [serial on the 
internet]. 2018 [cited 2020 Apr 11]; 8(2):258-68. Avaliable from: https://www5.bahiana.edu.br/index. php/fisioterapia/article/view/1962/1545

11. Nascimento CP, Morais KCS, Miranda VC, Ferreira JB. Síndrome de Burnout em Fisioterapeutas Intensivistas. Rev Pesq Fisio [serial on the internet]. 2017 [cited 2020 Apr 11]; 7(2):188-98. Avaliable from: https:// www5.bahiana.edu.br/index.php/fisioterapia/ article/view/1302

12. Silva RAD, Araújo B, Morais CCA, Campos SL, Andrade AD, Brandão DN. Síndrome de Burnout: realidade dos fisioterapeutas intensivistas? Fisioter Pesqui [serial on the internet]. 2018 [cited 2020 Nov 18]; 25(4):388-394. Avaliable from: http://www. scielo.br/scielo.php?script $=$ sci arttext \&pid $=\$ 1809$ $29502018000400388 \& \operatorname{lng}=$ en $\& n r m=$ is 0

13. Santos CLC, Barbosa GB, Nascimento DSS, Martins Junior DV, Nascimento Sobrinho CL. Prevalência de Síndrome da Estafa Profissional e fatores associados em fisioterapeutas intensivistas. Rev Pesq Fisio [serial on the internet]. 2018 [cited 2020 Apr 2020]; 8(3):336-344. Avaliable from: https://pesquisa. bvsalud.org/portal/resource/pt/biblio-915946

14. Maslach, C, Jackson S. The measurement of experienced Burnout. J 0ccup Behav [serial on the internet]. 1981 [cited 2020 Nov. 21]; 2(1):99-113. Avaliable from: https://onlinelibrary.wiley.com/ doi/abs/10.1002/job.4030020205

15. Barros DS, Tironi M0, Sobrinho CL, Neves FS, Bitencourt AG, Almeida AM, et al. Médicos plantonistas de unidade de terapia intensiva: perfil sócio-demográfico, condições de trabalho e fatores associados à síndrome de burnout. Rev Bras Ter Intensiva [serial on the internet]. 2008 [cited 2020 Dec 11]; 20(3):35-240. Avaliable from https://www.scielo.br/scielo.php?pid=S0103$\underline{507 \times 2008000300005 \& s c r i p t=s c i ~ a b s t r a c t \& t \operatorname{lng}=p t}$

16. Ferreira TS, Pereira PMC, Silva KR, Nobrega T0, Bastos VPD. Investigação da Síndrome de Burnout no ambiente de terapia intensiva. Cadernos ESP Ceará [serial on the internet]. 2019 [cited 2020 Apr. 9]; 13(1):19-26. Avaliable from: https://cadernos.esp.ce.gov.br/index.php/ cadernos/article/view/165/158

17. Alkimim CFC, Prado BMP, Carreiro DL, Coutinho LTM, Lima MRR, Martins AME, et al. Fatores associados à Síndrome de Burnout entre profissionais intensivistas de hospital universitário. Tempus, actas saúde colet [serial on the internet]. 2014 [cited 2020 Apr 9]; 8(4):157-76. Avaliable from: http://www.tempusactas.unb.br/index.php/tempus/ article/view/1590

18. Fumis RRL, Amarante GAJ, Nascimento AF, Junior JMV. Moral distress and its contribution to the development of burnout syndrome among critical care providers. Ann Intensive Care [serial on the internet]. 2017 [cited 2020 Apr 12]; 7:71. Avaliable from: https://www.ncbi.nlm.nih .gov/pmc/articles/PMC5479870/

19. Sacadura-Leite E, Sousa-Uva A, Ferreira S, Costa $\mathrm{PL}$, Passos AM. Condições de trabalho e exaustão emocional elevada em enfermeiros no ambiente hospitalar. Rev Bras Med Trab [serial on the internet]. 2019 [cited 2020 Apr 9]; 17(1):69-75. Avaliable from: http://www.rbmt.org.br/how-to-cite/422/pt-BR
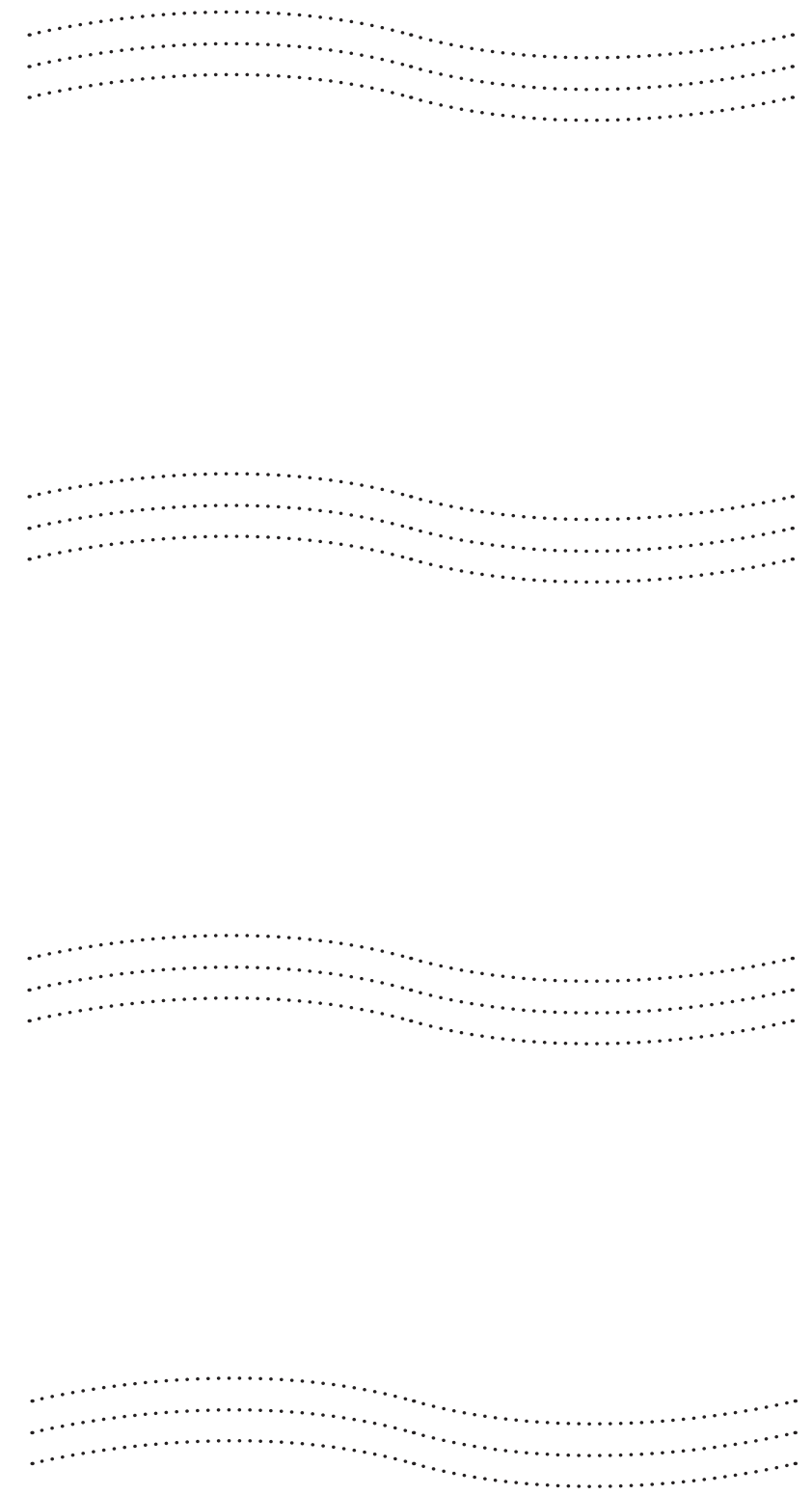

96 - SANARE (Sobral, Online). $2021 \mathrm{Jul-Dez;20(2):88-96}$ 